\title{
Grazing Incidence Pumping for Efficient X-ray Lasers
}

J. Dunn, R. Keenan, P. K. Patel, D. F. Price, R. F. Smith, V. N. Shlyaptsev

October 15, 2004

9th International Conference on X-ray Lasers Beijing, China

May 24, 2004 through May 28, 2004 
This document was prepared as an account of work sponsored by an agency of the United States Government. Neither the United States Government nor the University of California nor any of their employees, makes any warranty, express or implied, or assumes any legal liability or responsibility for the accuracy, completeness, or usefulness of any information, apparatus, product, or process disclosed, or represents that its use would not infringe privately owned rights. Reference herein to any specific commercial product, process, or service by trade name, trademark, manufacturer, or otherwise, does not necessarily constitute or imply its endorsement, recommendation, or favoring by the United States Government or the University of California. The views and opinions of authors expressed herein do not necessarily state or reflect those of the United States Government or the University of California, and shall not be used for advertising or product endorsement purposes. 


\title{
Grazing Incidence Pumping for Efficient X-ray Lasers
}

\author{
J. Dunn, R. Keenan, P.K. Patel, D.F. Price, R.F. Smith \\ Lawrence Livermore National Laboratory, Livermore, CA 94551, USA
}

\author{
V.N. Shlyaptsev \\ University of California, Davis, Livermore, CA 94551, USA
}

\begin{abstract}
We report progress in developing efficient pumping of laserdriven x-ray lasers that opens new possibilities for both high average power X-ray lasers as well as producing progressively shorter wavelength lasers. The new scheme of grazing incidence pumping (GRIP) is described. In essence, a chosen electron density region of a pre-formed plasma column, produced by a longer pulse at normal incidence onto a slab target, is selectively pumped by focusing the short pulse $\sim$ ps laser at a determined grazing incidence angle to the target. The controlled use of refraction of the pumping laser in the plasma results in several benefits: The pump laser path length is longer and there is an increase in the laser absorption in the gain region for creating a collisional Ni-like ion $\mathrm{x}$-ray laser. There is also an inherent travelling wave, close to $c$, that increases the overall pumping efficiency. The scheme requires careful tailoring of the pump and plasma conditions to the specific x-ray laser under investigation but the main advantage is a $3-30$ times reduction in the laser pump energy for mid- $Z$ materials. We report several examples of this new x-ray laser on two different laser systems. The first demonstrates a 10 $\mathrm{Hz}$ x-ray laser operating at $18.9 \mathrm{~nm}$ pumped with a total of $150 \mathrm{~mJ}$ of 800 $\mathrm{nm}$ wavelength from a Ti:Sapphire laser. The second case is shown where the COMET laser is used both at $527 \mathrm{~nm}$ and $1054 \mathrm{~nm}$ wavelength to pump higher $\mathrm{Z}$ materials with the goal of extending the wavelength regime of tabletop x-ray lasers below $10 \mathrm{~nm}$.
\end{abstract}

\section{Introduction}

Since the first demonstration of the laboratory x-ray laser (XRL) in 1984 on the LLNL Novette laser facility [1], there has been a world-wide effort to make these high brightness, short wavelength sources more efficient, more compact and to operate at a higher repetition rate. Until relatively recently, the mid-1990s, all research was conducted on very large, national class facilities requiring typically kilojoules of laser energy and at shot rates of a few per day. Some improvements, for example the pre-pulse technique, allowed approximately one order of magnitude reduction in the laser drive energy, $150 \mathrm{~J}$ 


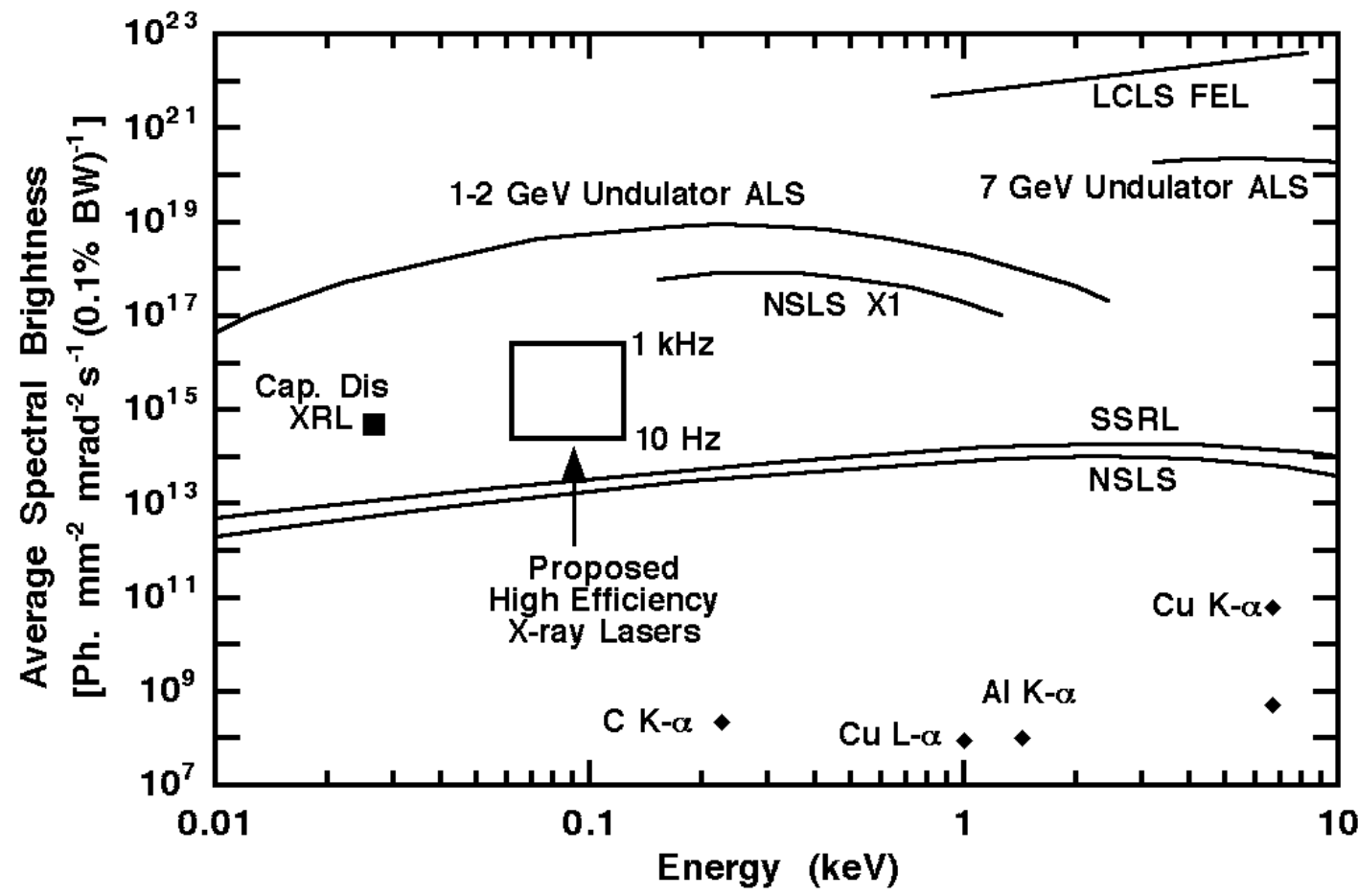

Figure 1. Comparison of average spectral brightness for various sources including present $3^{\text {rd }}$ generation synchrotrons, the proposed LCLS Free Electron Laser, and K- $\alpha$ sources. The proposed 100 fs -5 ps duration pumped high efficiency GRIP x-ray lasers of this work are shown as a box defined for $10-20 \mathrm{~nm}$ operation and $10 \mathrm{~Hz}-1 \mathrm{kHz}$ shot rates.

in 70 - 100 ps pulses, to drive a $13.9 \mathrm{~nm} \mathrm{x}$-ray laser in 1997 [2] and even as low as $30 \mathrm{~J}$ in 1999 [3].

Recently there have been a number of remarkable scientific breakthroughs that have led to the development of efficient, compact tabletop (occupying less than $10 \mathrm{~m}^{2}$ ) collisional excitation x-ray lasers with high output and a higher repetition rate. The picosecond laser-driven transient inversion lasers have allowed a dramatic reduction of pump energy to 5 - $10 \mathrm{~J}$ [4] while achieving saturated output for $14.7 \mathrm{~nm} \mathrm{x}$-rays at a repetition rate of 1 shot/4 minutes [5]. The fast capillary discharge technique developed at Colorado State University operates at a higher rate of $7 \mathrm{~Hz}$ for slightly longer $46.9 \mathrm{~nm}$ wavelength x-rays and is the first demonstration of a high-average power, tabletop system [6]. The capillary discharge scheme still remains the highest average power tabletop system with $10 \mathrm{~mW}$ of power at $46.9 \mathrm{~nm}$. These new short wavelength sources have ultra-high peak brightness $10^{24}-10^{25} \mathrm{ph} \mathrm{mm}^{-2} \mathrm{mrad}^{-2} \mathrm{~s}^{-1}(0.1 \% \mathrm{BW})^{-1}$ typically $4-5$ orders of magnitude higher than $3^{\text {rd }}$ generation synchrotrons. However, with the exception of the capillary discharge scheme shown in Fig. 1, most of the laser-pumped xray lasers until very recently had low average brightness.

A high repetition rate, high-average brightness laser-driven x-ray source would be very attractive if it could operate at shorter wavelengths $8-20 \mathrm{~nm}$. Near synchroton output levels with average brightness between the bending magnets and undulators, see Fig. 1, would be possible from a tabletop source. It should be kept in mind that this would be from a relatively inexpensive source (less than $\$ 1 \mathrm{M}$ ) when compared to the large expensive, central facilities like the $3^{\text {rd }}$ generation and planned $4^{\text {th }}$ generation synchrotron sources. High repetition rate $10 \mathrm{~Hz}$ laser-driven $\mathrm{x}$-ray lasers are beginning to be studied using the optical field ionization (OFI) process and have been demonstrated at 
$41.8 \mathrm{~nm}$ and $32.8 \mathrm{~nm}$ with less than $1 \mathrm{~J}$ pump energy/pulse [7]. It is a substantial technical and scientific challenge to operate x-ray lasers below $20 \mathrm{~nm}$ with the present high-repetition rate laser systems. The power required to drive an x-ray laser of wavelength, $\lambda$, typically scales $\sim \lambda^{-4}-\lambda^{-6}$. Furthermore, to operate our present picosecond-laser driven x-ray lasers [5] from $\sim 5 \mathrm{~J} / \mathrm{shot}$ to $150 \mathrm{~mJ} / \mathrm{shot}$ (about 30 times reduction) for $10 \mathrm{~Hz}$ operation for a small high-power laser requires a revolutionary step forward in efficiency. Nevertheless, the canonical x-ray conversion efficiency numbers, $\mathrm{x}$-ray laser energy out divided by laser pump energy in, are in the range of $10^{-7}-10^{-5}$. This suggests that there is still substantial room for improvement in pumping efficiency.

This paper addresses research activities using existing laser facilities within LLNL to study a new high-efficiency x-ray laser concept based on collisional excitation of Ni-like ion schemes. The grazing incidence pumping (GRIP) uses refraction of the short laser pump in a pre-formed plasma to deliver the laser energy into a specific electron density region where the inversion takes place. The benefits in achieving an efficient highrepetition rate $\mathrm{x}$-ray laser can be used for further development of shorter wavelength $\mathrm{x}$ ray lasers below $10 \mathrm{~nm}$ with modest $\sim 10 \mathrm{~J}$ facilities. It may also be possible to produce lasing close to the water-window with two orders of magnitude less energy than the $10 \mathrm{~kJ}$ required for the first $\mathrm{x}$-ray laser cellular imaging experiment conducted on Nova [8].

\section{Grazing Incidence Pumping Scheme for Mo $18.9 \mathrm{~nm}$ laser}

The GRIP scheme is proposed to dramatically improve the efficiency of laser-pumped $\mathrm{x}$ ray lasers below $20 \mathrm{~nm}$ for input energies of $\sim 150 \mathrm{~mJ}$. This is 30 times smaller than the energy of our picosecond laser-driven scheme that uses the well-established transverse pumping geometry for mid-Z Ni-like ions [5]. Simulations using the RADEX code indicate that while this is considerably more efficient than previous X-ray laser pumping, only a very small fraction of the absorbed laser energy, approximately $5-8 \%$, is deposited in the gain region of interest and pumping the inversion process. The recently developed diagnostic tools of near-field imaging of the $\mathrm{x}$-ray laser gain region and picosecond x-ray laser interferometry [9] have given new insight into the x-ray laser generation process. These results show a very narrow plasma gain region of $\sim 20 \mu \mathrm{m}$ width at a distance of $20-50 \mu \mathrm{m}$ from the target surface at an electron density of $\sim 10^{20}$ $\mathrm{cm}^{-3}$. Thus, in agreement with the predictions, much of the laser energy heats regions of the plasma that do not contribute to the observed lasing. In principle, longitudinal optical pumping, where the laser beam is focused at high intensity into the end of the plasma column, is appealing since it allows efficient traveling-wave heating and therefore copropagation with the x-ray laser pulse. Recently, a longitudinally pumped Ni-like Mo laser has been demonstrated [10]. However the considerable absorption, refraction, and

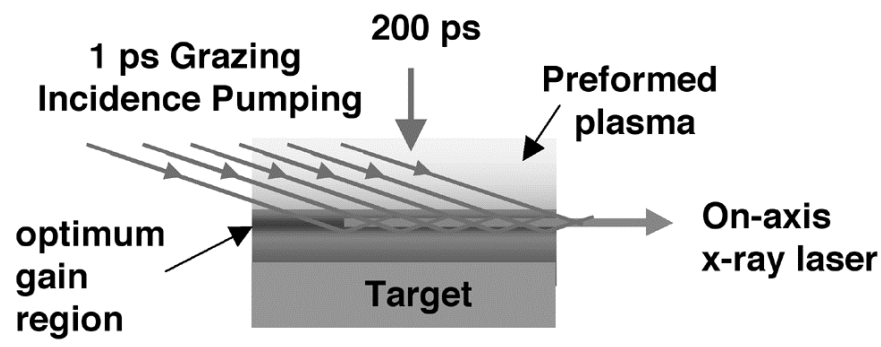

Figure 2. Schematic of grazing incidence pumping scheme. A pre-formed plasma is first produced in a flat target to generate the optimum gain region. Short pulse $1 \mathrm{ps}, 800 \mathrm{~nm}$ wavelength laser is incident at a grazing angle to strongly heat this region producing efficient on-axis $\mathrm{x}$-ray lasing. 
relativistic self-focusing of the high intensity short pulse optical pump make the longitudinally-pumped x-ray laser progressively difficult at higher electron densities.

However, utilisation of the high efficiency, inherent travelling wave for longitudinal schemes can be combined with some small component of transverse pumping to improve laser absorption in the desired density region with reduced detrimental refraction. We will call this grazing incidence pumping as proposed by Shlyaptsev and illustrated in Fig. 2 above. The main reason for the choice of the grazing angle is to use refraction of the pumping laser in a controlled way depending only on the predicted optimum electron density and wavelength of the laser pump. In the GRIP scheme the same two stage pumping is used as in the conventional transient scheme but with a significant difference. A long pulse, 200 ps in this case, focused on a Mo slab at normal incidence preforms a plasma column to create a tailored density profile. After a certain delay when the plasma conditions, particularly the electron density profile is correct, the short pulse is incident at a grazing angle to the target surface in a line focus. The short pulse beam is refracted in the plasma at a chosen electron density, well below critical, and is strongly absorbed. The required angle of incidence of the short pulse, measured relative to the target surface, is chosen to allow the laser pump to be refracted in the plasma. This is obtained from the equation for the refraction angle, $\phi_{r}$, simplified to $\phi_{r} \sim\left(n_{e o} / n_{e c}\right)^{1 / 2}$ where $n_{e o}$ is the maximum density within the gain region and $n_{e c}$ is the critical density for the optical pump [11]. This has several advantages that increase the laser coupling into the gain region. The grazing angle gives a longer path length to increase absorption within a specific plasma region so that a smaller active volume is pumped. Refraction causes the short pulse to turn back into the gain region thus allowing the laser pump to traverse this region twice. The short pulse beam has an inherent travelling wave very close to the speed of light. Also there is no restriction in the length of the plasma to be pumped. For these reasons, a sub-20 nm x-ray laser should be able to be generated on a small, highrepetition rate laser.

Initial RADEX simulations of the electron density profile 500 ps after the pre-pulse is fired, as well as electron temperature and absorbed laser power just after the short pulse is fired are shown in Fig. 3 for $800 \mathrm{~nm}$ pumping of a Mo target [11]. The predicted gain region is shown in the shaded area. Laser energy of $75-120 \mathrm{~mJ}, 200$ ps (FWHM) is incident at normal incidence at an intensity $\sim 2 \times 10^{11} \mathrm{~W} \mathrm{~cm}^{-2}$. The short pulse pumping conditions are chosen at a grazing angle of $13.6^{\circ}$ to couple strongly into $10^{20} \mathrm{~cm}^{-3}$ region. 
The pulse duration is $4 \mathrm{ps}$ (FWHM) with $64-100 \mathrm{~mJ}$ focused to $\sim 3-5 \times 10^{13} \mathrm{~W} \mathrm{~cm}^{-2}$. Some optimisation of the focal conditions is essential in order to maximise the deposition of laser energy into the gain region in the most efficient manner. The comparison between the GRIP and the transverse schemes show higher electron temperature $T_{e}$, Fig. 3(b), and higher absorbed power, Fig. 3(c) in the preferred gain region when the short pulse is incident at a grazing angle.

\section{Results for GRIP Ni-like Mo $10 \mathrm{~Hz}, 18.9 \mathrm{~nm}$ Laser Pumped with $150 \mathrm{~mJ}$}

The Ni-like Mo laser experiment is described in more detail in several places including these proceedings [12-14]. The laser pumping conditions at a repetition rate of $10 \mathrm{~Hz}$ were very similar to those described above. The long 200 ps pulse contained $70 \mathrm{~mJ}, 800$ $\mathrm{nm}$, and was focused at an intensity of $5 \times 10^{11} \mathrm{~W} \mathrm{~cm}^{-2}$ onto the Mo target at normal incidence in a line of $5 \mathrm{~mm} \times 15 \mu \mathrm{m}(\mathrm{FWHM})$. A maximum energy of $80 \mathrm{~mJ}$ in $1.5 \mathrm{ps}$, $800 \mathrm{~nm}$, was focused, at an intensity of $4 \times 10^{13} \mathrm{~W} \mathrm{~cm}^{-2}$. An on-axis parabola generated a very uniform line focus $4 \mathrm{~mm} \times \sim 25-30 \mu \mathrm{m}(\mathrm{FWHM})$ wide at the chosen $14^{\circ}$ angle with an intrinsic travelling wave velocity of 0.97c [14]. Careful adjustment of the pumping conditions were required to achieve the soft x-ray laser line. Reducing the laser energy by more than $10 \%$ in each beam resulted in a strong fall in x-ray laser intensity. This indicated that we were pumping close to the threshold for $18.9 \mathrm{~nm}$ lasing on the $4 d-$ $4 p$ line. The optimised pump conditions were found to be a $1.5 \mathrm{ps}$ short pulse delayed by $500 \mathrm{ps}$ after the peak of the long pulse. Lasing was also observed for pumping from 100 fs to 6 ps short pulse durations. High gains of $\sim 60 \mathrm{~cm}^{-1}$ were determined with strong output for target lengths up to a maximum of $4 \mathrm{~mm}$. The gain saturation regime was achieved based on the intensity versus length measurements $[13,14]$.

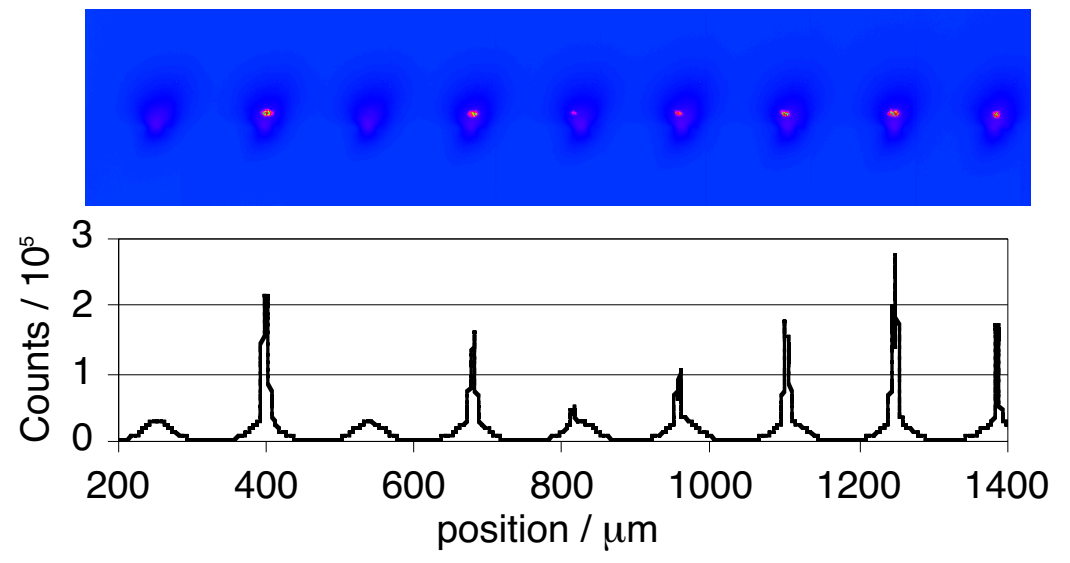

Figure 4. Near-field imaging (using multilayer optics) of the GRIP Ni-like Mo $18.9 \mathrm{~nm}$ x-ray laser. The $\mathrm{x}$-ray laser is pumped at high repetition rate during the CCD camera readout. Lower image is lineout through near-field intensity.

An on-axis soft x-ray Mo:Si multilayer-coated mirror imaging system, consisting of a $12.5 \mathrm{~cm}$ focal length mirror in combination with a $45^{\circ}$ mirror, was used to image the near-field pattern of the $18.9 \mathrm{~nm}$ Mo x-ray laser at $17 \times$ magnification onto a backilluminated CCD. Many laser near-field image x-ray laser shots could be recorded in one $\mathrm{CCD}$ frame by synchronising the repetition rate of the laser with the CCD readout. Figure 4 shows a sequence of frames for the x-ray laser plasma imaged on-axis running at high repetition rate. A variation in the XRL output was observed which was attributed to a vibration-induced low frequency oscillation in the short pulse line focus. The overlap 
between the two line foci separates on some shots by no more than one width $\sim 20 \mu \mathrm{m}$. As the short pulse line focus moves back towards the center of the preformed plasma, 5 images on the right hand-side, the XRL output increases to a maximum. This allows us to test the sensitivity of the line focus overlap important for high repetition sources. The near-field $\mathrm{x}$-ray laser beam size was less than $\sim 9 \times 20 \mu \mathrm{m}^{2}$ and showed some structure present in the image. Initial estimates of the x-ray laser energy were in the range of 10 $20 \mathrm{~nJ}$ but could be higher. This will be reported in a Ni-like Mo characterisation paper in detail at a later date.

(a)

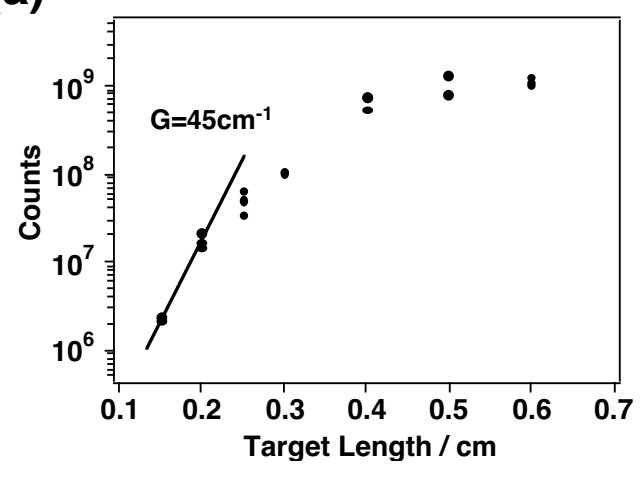

(c)

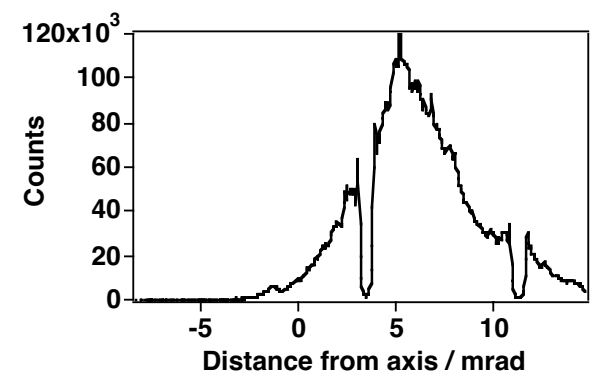

(b)

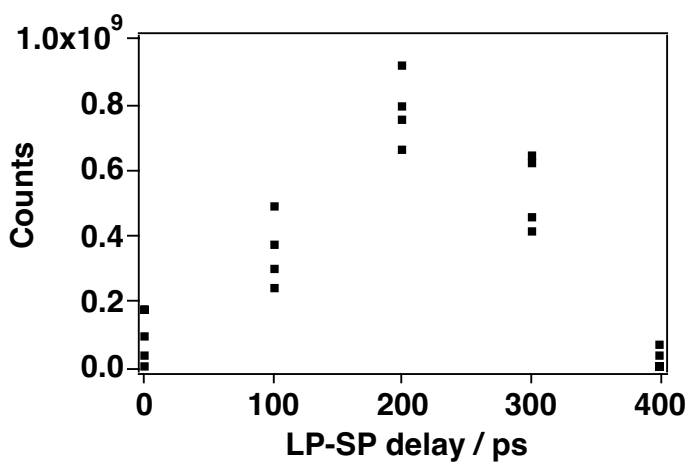

(d)

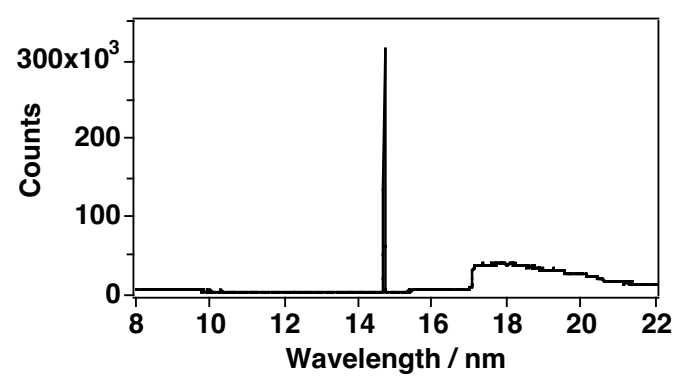

Figure 5. GRIP experiments conducted on the COMET laser using $1 \omega \mathrm{LP} / 2 \omega \mathrm{SP}$ pumping on the Nilike Pd $14.7 \mathrm{~nm}$ X-ray laser. (a) X-ray laser $14.7 \mathrm{~nm}$ output intensity plotted as a function of Pd target length indicating gains of $45 \mathrm{~cm}^{-1}$. (b) Optimum delay between the laser pulses was found to be $\Delta t$ of 200 ps. (c) Horizontal beam deflection for $14.7 \mathrm{~nm}$ x-ray laser. (d) X-ray laser spectrum filtered by 200 $\mathrm{nm} \mathrm{Al}$ filter showing very strong output in the Ni-like Pd line pumped by $2 \mathrm{~J}$ energy.

\section{GRIP Scheme Implementation on COMET}

The first Ti:Sapphire, $800 \mathrm{~nm}, 10 \mathrm{~Hz}$ GRIP experiments reported in the previous section were working at the limits of the available laser pump energy. More energy to produce a larger line focus, both in width and length for both beams, was required. So the GRIP experiments were transferred to COMET to test the pumping principle with more energy at both $1054 \mathrm{~nm}$ and $527 \mathrm{~nm}$ wavelengths. It is not possible to summarise all of the parameters studied here but the technique was checked and lasing was achieved for numerous mid-Z Ni-like ions as short as $12 \mathrm{~nm}$ with $\sim 2$ - $4 \mathrm{~J}$ pump energy. 
One example is described where a $1 \omega,(1054 \mathrm{~nm}) 600 \mathrm{ps}$ pulse was used in combination with a $2 \omega,(527 \mathrm{~nm}) 1.5 \mathrm{ps}$ short pulse. Both beams were focused to $\sim 7-8$ $\mathrm{mm} \times 40 \mu \mathrm{m}(\mathrm{FWHM})$. The Ni-like Pd laser was chosen for this study. The optimum electron density for the turning point was chosen to be $1.2 \times 10^{20} \mathrm{~cm}^{-3}$ which required a pumping angle of $10^{\circ}$. The laser energy was approximately $1.2 \mathrm{~J}-1.3 \mathrm{~J}$ in each beam. After some optimisation it was found that there was very strong output available in the $\mathrm{x}-$ ray laser. The results are shown in Fig. 5. The optimum delay was found to be $200 \mathrm{ps}$ between the pulses with a wider window than the previous Mo results [13, 14], Fig. 5(b). The gain was determined to be $45 \mathrm{~cm}^{-1}$ and the laser goes into saturation with $g L \sim 14$ at $4 \mathrm{~mm}$, see Fig 5(a). The deflection angle of the $14.7 \mathrm{~nm}$ x-ray laser as it leaves the plasma was $5 \mathrm{mrad}$ away from the target, Fig. 5 (c). Although the larger focal geometries require $\sim 10 \times$ larger pumping energy they appear to generate more than $100 \times$ higher $x$-ray laser output. For targets above $4 \mathrm{~mm}$ in length, the output had to be attenuated using $200 \mathrm{~nm}$ Al filters to prevent the CCD from saturating on the on-axis spectrometer, Fig. 5(d). Initial comparisons suggest that the GRIP XRL output in a $6 \mathrm{~mm}$ target are comparable with a $1.2 \mathrm{~cm}$ target pumped transversely with $\sim 5 \mathrm{~J}$ in the short pulse. This indicates that a reduction of $3-4 \times$ in short pulse pump energy has been achieved for the same $x$-ray laser output. These are only the second set of experiments and further improvements will be possible as more groups employ the grazing incidence pumping scheme.

\section{Summary and Future Directions}

In summary the grazing incidence $\mathrm{x}$-ray laser scheme shows substantial promise for producing higher efficiency coupling of the laser pump energy into the plasma gain region. The underlying principle is the controlled use of refraction of the pump beam to selectively pump a specific electron density and gain volume. We have shown that it can work with as little as $150 \mathrm{~mJ}$ pump energy for a $10 \mathrm{~Hz}$ Ni-like Mo $18.9 \mathrm{~nm}$ x-ray laser. It has been driven into saturation though there is still more potential to extract higher x-ray laser output energy. Additional results demonstrating the GRIP pumping are reported by the Colorado State University group [15]. The GRIP experiments on COMET show that the scheme appears to be universally applicable to many pumping configurations and pulse lengths. It can operate on numerous higher $\mathrm{Z}$ materials at different angles and different pump wavelengths though in each case some optimisation is likely to be required. Increasing the laser pump energy to $\sim 1 \mathrm{~J} /$ pulse gives substantial output for the mid-Z Ni-like ions X-ray lasers. There is also the prospect of extending lasing to shorter wavelengths with this technique to re-visit water-window x-ray lasers for biological applications.

\section{Acknowledgments}

The continued support and encouragement of Al Osterheld is greatly appreciated. The authors would like to thank Joe Nilsen for the use of the multilayer optics in part of this work. Work performed under the auspices of the US Department of Energy by the University of California Lawrence Livermore National Laboratory under Contract No. W-7405-Eng-48 and in part by funding from the National Science Foundation through the Center for Biophotonics, an NSF Science and Technology Center, managed by the University of California, Davis, under Cooperative Agreement No. PHY 0120999.

\section{References}

[1] D. Matthews et al., Phys. Rev. Lett. 54, 110 (1985).

[2] J. Nilsen et al., Phys. Rev. A 48, 4682 (1993); J. Zhang et al., Phys. Rev. Lett. 78, 3856 (1997). 
[3] R. Tommasini et al., Phys. Rev. A 59, 1577 (1999).

[4] P. Nickles et al., Phys. Rev. Lett. 78, 2748 (1997); J. Dunn et al., ibid. 80(13), 2825 (1998).

[5] J. Dunn et al., ibid. 84, 4834 (2000).

[6] B.R. Benware, C.D. Macchietto, C.H. Moreno and J.J. Rocca, Phys. Rev. Lett. 81, 5804, (1998).

[7] S. Sebban et al., Phys. Rev. Lett. 86, 3004 (2001); S. Sebban et al., Phys. Rev. Lett. 89, 253901 1-4 (2002).

[8] L.B. Da Silva et al., Science 258, 269 (1992).

[9] R.F. Smith et al., Phys. Rev. Lett, 89, 065004-1 (2002); R.F. Smith et al., Soft x-ray lasers and Applications V, SPIE Int. Soc. Opt. Eng. Proc, vol. 5197, ed. E.E. Fill and S. Suckewer, 155-167 (2003).

[10] T. Ozaki et al., Phys. Rev. Lett. 89, 253902-1 (2002).

[11] V.N. Shlyaptsev et al., Soft x-ray lasers and Applications V, SPIE Int. Soc. Opt. Eng. Proc, vol. 5197, ed. E.E. Fill and S. Suckewer, 221 - 228 (2003).

[12] R. Keenan et al., Soft x-ray lasers and Applications V, ibid., 213 - 220 (2003).

[13] R. Keenan et al., "Grazing Incidence Pumping for Efficient X-ray Lasers", in these proceedings (2004).

[14] R. Keenan et al., "High Repetition Rate Grazing Incidence Pumped X-ray Laser Operating at $18.9 \mathrm{~nm}$ ", submitted to Phys. Rev. Lett. (2004).

[15] J.J. Rocca et al., "Demonstration of High Repeition Rate Desk-Top and Table-Top Soft X-ray Lasers", in these proceedings (2004). 